\title{
Linear reinforcement learning: Flexible reuse of computation in planning, grid fields, and cognitive control
}

\author{
Payam Piray* and Nathaniel D. Daw \\ Princeton Neuroscience Institute, Princeton University \\ Corresponding author and lead contact: ppiray@princeton.edu
}

\begin{abstract}
It is thought that the brain's judicious allocation and reuse of computation underlies our ability to plan flexibly, but also failures to do so as in habits and compulsion. Yet we lack a complete, realistic account of either. Building on control engineering, we introduce a new model for decision making in the brain that reuses a temporally abstracted map of future events to enable biologically-realistic, flexible choice at the expense of specific, quantifiable biases. It replaces the classic nonlinear, model-based optimization with a linear approximation that softly maximizes around (and is weakly biased toward) a learned default policy. This solution exposes connections between seemingly disparate phenomena across behavioral neuroscience, notably flexible replanning with biases and cognitive control. It also gives new insight into how the brain can represent maps of long-distance contingencies stably and componentially, as in entorhinal response fields, and exploit them to guide choice even under changing goals.
\end{abstract}




\section{Introduction}

A key insight from reinforcement learning models is that humans' ability flexibly to plan new actions - and also our failures sometimes to do so in healthy habits and disorders of compulsion - can be understood in terms of the brain's ability to reuse previous computations(Daw and Dayan, 2014; Daw et al., 2005; Keramati et al., 2011; Russek et al., 2017; Stachenfeld et al., 2017). Exhaustive, "model-based" computation of action values is time-consuming; thus, it is deployed only selectively (such as early in learning a new task), and when possible, the brain instead bases choices on previously learned ("cached," "model-free") decision variables(Daw et al., 2005; Keramati et al., 2011). This strategy saves computation, but gives rise to slips of action when cached values are out-of-date.

However, while the basic concept of adaptive recomputation seems promising, this class of models - even augmented with refinements such as prioritized replay, partial evaluation, and the successor representation - has so far failed fully to account either for the brain's flexibility or its inflexibility(Momennejad et al., 2017; Russek et al., 2017). For flexibility, we still lack a tractable and neurally plausible account how the brain accomplishes the behaviors associated with model-based planning. Conversely, the reuse of completely formed action preferences can explain extreme examples of habits (such as a rat persistently working for food it doesn't want), but fails fully to explain how and when these tendencies can be overridden, and also many subtler, graded or probabilistic response tendencies, such as Pavlovian biases or varying error rates in cognitive control tasks.

Here, we introduce a new model that more nimbly reuses precursors of decision variables, so as to enable a flexible, tractable approximation to planning that is also characterized by specific, graded biases. The model's flexibility and inflexibility (and its ability to explain a number of other hitherto separate issues in decision neuroscience) are all rooted in a new approach to a core issue in choice. In particular, we argue that the central computational challenge in sequential decision tasks is that the optimal decision at every timepoint depends on the optimal decision at the next timepoint, and so on. In a maze, for instance, the value of going left or right now depends on which turn you make at the subsequent junction, and similarly thereafter; so, figuring out what is the best action now requires, simultaneously, also figuring out what are the best choices at all possible steps down the line. This interdependence between actions is a direct consequence of the natural definition of the objective function in this setting (i.e., the Bellman equation(Bellman, 1957)), and this greatly complicates planning, replanning, task transfer, and temporal abstraction in both artificial intelligence and biological settings(Sutton and Barto, 2018).

How, then, can the brain produce flexible behavior? Humans and animals can solve certain replanning tasks, such as reward devaluation and shortcuts, which require generating new action plans on the fly (Behrens et al., 2018; Dickinson and Balleine, 2002; Momennejad et al., 2017; Tolman, 1948; Wimmer and Shohamy, 2012). It has been argued that the brain does so by some variant of model-based planning; that is, solving the Bellman equation directly by iterative search(Daw et al., 2005; Keramati et al., 2011). However, we lack a biologically realistic account how this is implemented in the brain(Daw and Dayan, 2014); indeed, because of the interdependence of optimal actions, exhaustive search (e.g., implemented by neural replay(Mattar and Daw, 2018)) seems infeasible for most real-world tasks due to the 
exponentially growing number of future actions that must each be, iteratively and nonlinearly optimized. It has thus also been suggested that the brain employs various shortcuts that rely on reusing previously computed ("cached") quantities, notably model-free long-run values(Huys et al., 2015; Keramati et al., 2016).

One such proposal, which is perhaps the most promising step toward a neurally realistic planning algorithm is the successor representation (SR)(Dayan, 1993), which by leveraging cached expectations about which states will be visited in future, can efficiently solve a subset of tasks traditionally associated with model-based planning(Momennejad et al., 2017; Russek et al., 2017). However, it simply assumes away the key interdependent optimization problem by evaluating actions under a fixed choice policy (implied by the stored state expectancies) for future steps. This policy-dependence makes the model incapable of explaining how the brain can solve other replanning tasks, in which manipulations also affect future choices(Lehnert et al., 2017; Russek et al., 2017). In general, the precomputed information stored by the SR is only useful for replanning when the newly replanned policy remains similar to the old one: For instance, a change in goals implies a new optimal policy that visits a different set of states, and a different SR is then required to compute it. This is just one instance of a general problem that plagues attempts to simplify planning by temporal abstraction (e.g., chunking steps(Botvinick et al., 2009; Dezfouli and Balleine, 2012)), again due to the interdependence of optimal actions: if my goals change, the optimal action at future steps (and, hence, the relevant chunked long-run trajectories) likely also change.

Here, we adopt and build on recent advances in the field of control engineering(Kappen, 2005; Todorov, $2007,2009)$ to propose a new model for decision making in the brain that can efficiently solve for an approximation to the optimal policy, jointly across all choices at once. It does so by relying on a precomputed, temporally abstract map of long-run state expectancies similar to the SR, but one which is, crucially, stable and useful even under changes in the current goals and the decision policy they imply. The model, termed linear RL, provides a common framework for understanding different aspects of animals' cognitive abilities, particularly flexible planning and replanning using these temporally abstract maps, but also biases in cognitive control and Pavlovian influences on decision making, which arise directly from the strategy of reuse.

The model is based on a reformulation of the classical decision problem, which makes "soft" assumptions about the future policy (in the form of a stochastic action distribution), and introduces an additional cost for decision policies which deviate from this baseline. This can be viewed as an approximation to the classic problem, where soft, cost-dependent optimization around the default policy stands in for exact optimization of the action at each successor state. Crucially, the form of the costs allows the modified value function to be solved analytically using inexpensive and biologically plausible linear operations. In particular, the optimal value of any state under any set of goals depends on a weighted average of the long-run occupancies of future states that are expected under the default policy. Therefore, we propose that the brain stores a map of these long-run state expectancies across all states (the default representation, or DR), which gives a metric of closeness of states under the default policy. Because the DR depends only on the default policy, and can be used to compute a new optimal policy for arbitrary goals, the model can solve a large class of replanning tasks, including ones that defeat the SR. 
Our novel modeling approach also addresses a number of seemingly distinct questions. First, the stability of the DR across tasks makes it a candidate for understanding the role in decision-making of multiscale, temporally abstract representations in the brain, notably grid cells in the medial entorhinal cortex. These cells show regular grid-like firing patterns over space, at a range of frequencies, and have been argued to represent something akin to a Fourier-domain map of task space (e.g., the eigenvectors of the SR, equivalent to the graph Laplacian(Gustafson and Daw, 2011; Stachenfeld et al., 2017)), and could provide some sort of mechanism for spatial(Hafting et al., 2005) and mental navigation(Behrens et al., 2018; Constantinescu et al., 2016; Whittington et al., 2019). However, it has been unclear how this and similar long-run temporal abstractions are actually useful for planning or navigation, because as mentioned longrun (low-frequency) expectancies over task space are not stable across tasks due to the interdependence of policy, goals, and trajectories(Mahadevan, 2012; Mahadevan and Maggioni, 2007). For instance, because the SR only predicts accurately under the training policy, to be even marginally useful for replanning the SR theory predicts grid fields must continually change to reflect updated successor state predictions as the animal's choice policy evolves, which is inconsistent with evidence(Carpenter et al., 2015; Derdikman et al., 2009; Sanguinetti-Scheck and Brecht, 2019). The linear RL theory clarifies how the DR, a stable and globally useful long-run map under a fixed default policy, can serve flexible planning. Our theory also provides a new account for updating maps in situations which actually do require modification - notably, the introduction of barriers. We show how these give rise to additional, separable basis functions in the corresponding DR, which we associate with a distinct class of entorhinal response fields, the border cells. This aspect of the work goes some way toward delivering on the promise of such response as part of a reusable, componential code for cognitive maps(Behrens et al., 2018; Constantinescu et al., 2016).

Finally, linear RL addresses the flip side of how the brain can be so flexible: why, in some cases it is inflexible. We suggest that this is simply another aspect of the same mechanisms used to enable flexible planning. While it has long been suggested that fully model-free learning in the brain might account for extreme cases of goal-inconsistent habits (e.g., animals persistently working for food when not hungry(Daw et al., 2005)), there are many other phenomena which appear as more graded or occasional biases, such as Stroop effects, Pavlovian tendencies, slips of action(de Wit et al., 2007), and more sporadic failures of participants to solve replanning tasks(Momennejad et al., 2017). The default policy and cost term introduced to make linear RL tractable offers a natural explanation for these tendencies, quantifies in units of common-currency reward how costly it is to overcome them in different circumstances, and relatedly offers a novel rationale and explanation for a classic problem in cognitive control: the source of the apparent costs of "control-demanding" actions.

Despite its simplicity, the linear RL model accounts for a diverse range of problems across different areas of behavioral neuroscience. In the reminder of this article, we present a series of simulation experiments that demonstrate that the theory provides i) a biologically-realistic, efficient and flexible account of decision making; ii) a novel understanding of entorhinal grid code that explains its role in flexible planning, navigation and inference; iii) an understanding of cognitive control that naturally links it to other aspects of decision systems; and iv) a normative understanding of Pavlovian-instrumental transfer (PIT). 


\section{Results}

\section{The Model}

In Markov decision tasks, like mazes or video games, the agent visits a series of states $s$, and at each they receive some reward or punishment $r$ and choose among a set of available actions $a$, which then affects which state they visit next(Sutton and Barto, 2018). The objective in this setting is typically to maximize the expected sum of future rewards, called the 'value' function. Formally, the optimal value $\bar{v}^{*}$ of some state is given by the sum of future rewards, as a series of nested expectations:

$$
\bar{v}^{*}\left(s_{t}\right)=r\left(s_{t}\right)+\max _{a_{t}} \sum_{s_{t+1}} P\left(s_{t+1} \mid s_{t}, a_{t}\right)\left[r\left(s_{t+1}\right)+\max _{a_{t+1}} \sum_{s_{t+2}} P\left(s_{t+2} \mid s_{t+1}, a_{t+1}\right)\left[r\left(s_{t+2}\right)+\cdots\right]\right]
$$

or equivalently in recursive form by the Bellman equation (Bellman, 1957):

$$
\bar{v}^{*}\left(s_{t}\right)=r\left(s_{t}\right)+\max _{a_{t}} \sum_{s_{t+1}} P\left(s_{t+1} \mid s_{t}, a_{t}\right) \bar{v}^{*}\left(s_{t+1}\right)
$$

Across all states, this results in a set of interdependent optimization problems, which can be solved, for instance, by iterative search through the tree of future states, computing the maximizing action at each step(Sutton and Barto, 2018). However, in realistic tasks with large state spaces, this iterative, nonlinear computation may be intractable.

Note that prediction can be used for action choice or computing an action selection policy: once we have computed $\bar{v}^{*}$ (the optimal future reward available from each state), we can just compare it across actions to find the best action in any particular state and form a policy, $\pi^{*}$; for instance, we can evaluate the max in equation (1) for any state, plugging in the optimal values of successor states without further iteration. However, note also that this depends on having already found the maximizing action at other states down the line, since $\bar{v}^{*}$ depends, recursively, on which actions are taken later, and this in turn depends on the assignment of rewards to states (e.g., the agent's goals).

If we instead assumed that we were going to follow some given, not necessarily optimal, action selection policy $\pi$ at each subsequent state (say, choosing randomly), then equation (1) would be replaced by a simple set of linear equations (eliminating the nonlinear function "max" at each step) and relatively easily solvable. This observation is the basis of the SR model(Dayan, 1993; Momennejad et al., 2017; Russek et al., 2017; Stachenfeld et al., 2017), which computes values as

$$
\overline{\mathbf{v}}^{\pi}=\mathbf{S}^{\pi} \mathbf{r}
$$

where (in matrix-vector form) $\overline{\mathbf{v}}^{\pi}$ is a vector of long-run state values under the policy $\pi ; \mathbf{r}$ a vector of state rewards; and $\mathbf{S}^{\pi}$ a matrix measuring which subsequent states one is likely to visit in the long run following a visit to any starting state: importantly, assuming that all choices are made following policy $\pi$. However, although this allows us to find the value of following policy $\pi$, this does not directly reveal how to choose optimally. For instance, plugging these values into equation (1) won't produce optimal choices, since $\overline{\mathbf{v}}^{\pi}$ (the value of choosing according to $\pi$ in the future) in general does not equal the value, $\overline{\mathbf{v}}^{*}$, of choosing 
optimally. The only way to find the latter using equation (2) is by iteratively re-solving the equation to repeatedly update $\pi$ and $\mathbf{S}$ until they eventually converge to $\pi^{*}$, i.e., the classic policy iteration algorithm.

It has recently been shown that a change in the formulation of this problem, which we refer to as linear $R L$, greatly simplifies the Bellman equation(Kappen, 2005; Todorov, 2007, 2009). To see this, we first assume a one-to-one, deterministic correspondence between actions and successor states (i.e., for every state $s^{\prime}$ reachable in one step from some $s$, assume there is a corresponding action $a$ for which $P\left(s^{\prime} \mid s, a\right)=1$, which is simply denoted by its destination, $\left.s^{\prime}\right)$. This fits many problems with fully controllable, deterministic dynamics, such as spatial navigation (where for each adjacent location, there is a corresponding action taking you there). Second, linear RL seeks to optimize not a discrete choice of successor state (action), but a stochastic probability distribution $\pi$ over it(Todorov, 2007, 2009). Finally, it redefines the value function to include not just the one-step rewards $r$ but also at each step a new penalty(Kappen, 2005; Todorov, 2007, 2009), called a "control cost," $\operatorname{KL}\left(\pi|| \pi^{d}\right)$, which is increasing in the dissimilarity (KL divergence) between the chosen distribution $\pi$ and some default distribution, $\pi^{d}$.

Linear RL is most naturally a formalism for modeling tasks in which there are some default dynamics (e.g., a rocket in a gravitational field) and costly actions to modify them (e.g., firing thrusters burning different amounts of fuel). Alternatively, here we view it as an approximation to the original value function, where the additional penalty terms modify the original problem to a related one that can be more efficiently solved. This is because linear RL deals with the problem of the interdependence of the optimal actions across states(Kappen, 2005; Todorov, 2007, 2009): the default policy $\pi^{d}$ represents a set of soft assumptions about which actions will be taken later, which are optimized into an optimal stochastic distribution $\pi^{*}$ that is approximately representative of the optimal (deterministic) subsequent choices in the original problem.

Efficient solution is possible because, substituting the penalized rewards into the Bellman equation, the optimal value function is now given by a non-recursive, linear equation(Todorov, 2007, 2009):

$$
\exp \left(\mathbf{v}^{*}\right)=\mathbf{M P} \exp (\mathbf{r})
$$

such as can be computed by a single layer of a simple, linear neural network. Here, $\mathbf{v}^{*}$ is a vector of the optimal values (now defined as maximizing cumulative reward minus control cost) for each state; $\mathbf{r}$ is a vector of rewards at a set of "terminal" states (i.e., various possible goals); $\mathbf{P}$ is a matrix containing the probability of reaching each goal state from each other, nonterminal, state; and the key matrix $\mathbf{M}$, which we call the default representation (DR), measures the closeness of each nonterminal state to each other nonterminal state (in terms of expected aggregate cost to all future visits) under the default policy. This is similar to the SR ( $\mathbf{S}^{\pi}$, equation (2)), except that it is for the optimal values $\mathbf{v}^{*}$ (not the on-policy values $\mathbf{v}^{\pi}$ ), and $\mathbf{v}^{*}$ is systematically related to optimal values as defined in the original problem $\left(\overline{\mathbf{v}}^{*}\right.$, Eq. 1$)$, with the difference being the additional penalties for deviation from the default policy. But these exert only a soft bias in $\pi^{*}$ toward $\pi^{d}$, which furthermore vanishes altogether in an appropriate limit (see Methods). Thus, while $\mathbf{M}$ does depend on the default policy $\pi^{d}$, it is stable over changes in goals and independent from $\pi^{*}$ in the sense that it can usefully find optimized policies $\pi^{*}$ even when these are far from $\pi^{d}$; in comparison, $\mathbf{v}^{\pi}$ (computed from the SR: $\mathbf{S}^{\pi}$ ) is only a useful aproximation to $\mathbf{v}^{*}$ (and thus only helpful in 
finding a new $\pi^{*}$ ) when the SR's learned policy $\pi$ is near the target policy $\pi^{*}$. Effectively, linear RL works by introducing a smooth approximation of the "max" in equation (1), since the log-average-exp (with the average here taken with respect to the default distribution, $\pi^{d}$ ) of a set of values approximates the maximum. The control costs, then, simply capture the difference between the original solution and the smooth approximate one.

\section{Model Performance}

The optimized policy in this model balances expected reward with control cost, and is generally stochastic rather than deterministic, like a softmax function (Fig 1a-b). We evaluated the performance of linear RL as an approximation to exact solution by considering a difficult, 7-level decision tree task in which each state has two possible successors, a set of costs are assigned randomly at each state, and the goal is to find the cheapest path to the bottom. We conducted a series of simulations, comparing linear RL with a set of benchmarks: exact (model-based) solution, and a set of approximate model-based RL agents(Keramati et al., 2016) that optimally evaluate the tree up to a certain depth, then "prune" the recursion at that leaf by substituting the exact average value over the remaining subtree (Fig 1c; in the one-step case this is equivalent to the SR under the random walk policy). For linear RL, the default policy was taken as a uniform distribution over possible successor states. Linear RL achieved near-optimal average costs (Fig 1d).

a

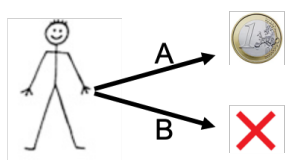

C

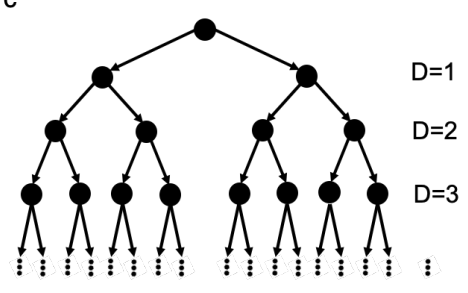

b

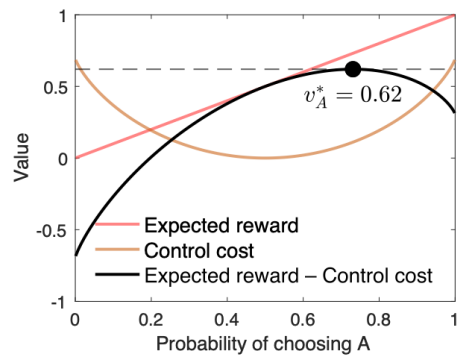

d

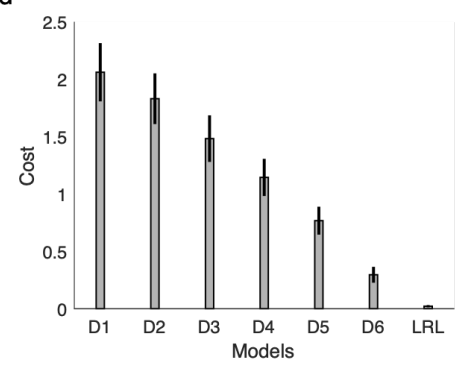

Fig 1. The linear RL model. a-b) the model optimizes the decision policy by considering the reward and the control cost, which is defined as the KL divergence between the decision policy and a default policy. Assuming an unbiased (uniform) distribution as the default policy, the optimal decision policy balances the expected reward with the control cost. Although the expected reward is maximum when probability of choosing $A$ is close to 1 (and therefore probability of choosing $B$ is about zero), this decision policy has maximum control cost due to its substantial deviation from the default policy. The optimal value instead maximized expected reward minus the control cost, which here occurs when probability of choosing $A$ is 0.73 . c-d) The model accurately approximates optimal choice. We compared its 
performance on a 7-level decision tree task (with random one-step costs at each state) to 6 pruned model-based RL algorithms, which evaluate the task to a certain depth $(D=1, . ., 6$; D7 is optimal; D1 is equivalent to the successor representation for the random walk policy) and use average values at the leaves. Linear RL (LRL) achieved near-optimal average costs ( $y$-axis is additional cost relative to the optimum). Local costs of all states were randomly chosen in the range of 0 to 10 , and simulations were repeated 100 times. Mean and standard error across all simulations are plotted.

An important aspect of linear RL is that the DR, M, reflects the structure of the task (including the distances between all the nonterminal states under the default policy) in a way that facilitates finding the optimal values, but is independent of the goal values $\mathbf{r}$, and the resulting optimized value and policy (Fig 2). Therefore, by computing or learning the DR once, the model is able to re-plan under any change in the value of the goals (see below) and also (with some additional computation to efficiently add an additional terminal goal state, see Methods), plan toward any new goal with minimal further computation (Fig $2 \mathrm{~b}$ c). In the case of spatial tasks, this corresponds to finding the shortest path from any state to any goal state. In fact, our simulation analysis in a maze environment revealed that linear RL efficiently finds the shortest path between every two states in the maze (Fig $2 \mathrm{~d}$ ).

a

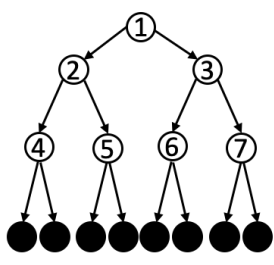

b

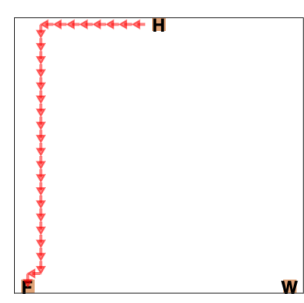

d

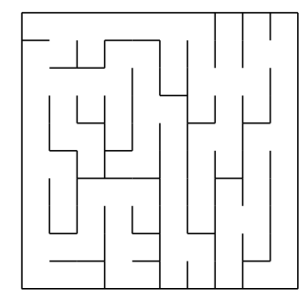

DR

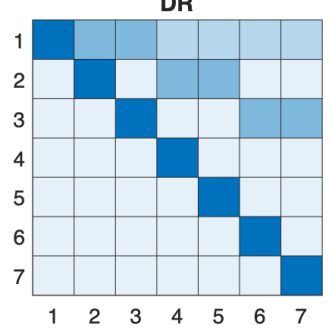

c
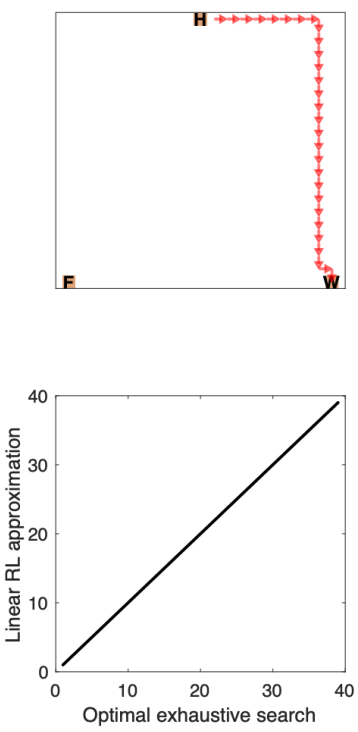

Fig 2. Default representation. a) The DR corresponding to a three-level decision tree task is shown. Each row of the DR represents weighted future expectancies starting from the corresponding state and following the default policy. Therefore, the DR is independent of the goals and optimized policy. b-c) The optimized path for planning from home $(H)$ to the food $(F)$ state is computed based on the DR. The linear RL model is efficient because the same DR is sufficient for planning towrds a new goal, such as the water (W) state. d) The path between every two states in a 10by-10 maze environment (d) computed by linear RL exactly matches the optimal (shortest) path computed by exhaustive search. The DR has been computed once and reused (in combination with techinuqes from matrix identities) to compute each optimal path.

\section{Replanning}

In both artificial intelligence, and psychology and biology, a key test of efficient decision making is how an agent is able to transfer knowledge from one task to another. For instance, many tasks from neuroscience test whether organisms are able, without extensive retraining, to adjust their choices following a change 
in the rewards or goals ("revaluation," "devaluation," "latent learning") or transition map ("shortcut," "detour") of a previously learned task(Dickinson and Balleine, 2002; Momennejad et al., 2017; Tolman, 1948; Wimmer and Shohamy, 2012). We explored the ability of linear RL for solving these types of replanning problems (Fig 3). Importantly, the model is able to solve one class of these problems - those involving revaluation of goals - efficiently, as the DR can be used, unmodified, to solve any new problem. This corresponds to simply changing $\mathbf{r}$ in Eq. 3, and computing new values.

First, we confirmed that linear RL is able to solve a version of Tolman's latent learning task (Fig 3a), a revaluation task in which rats were first trained to forage freely in a maze with two rewarding end-boxes, but then were shocked in one of the end-boxes to reduce its value(Tolman and Gleitman, 1949). This manipulation defeats model-free RL algorithms like temporal difference learning, because they must experience trajectories leading from the choice to the devalued box to update previously learned longrun value or policy estimates(Daw et al., 2005). In contrast, rats are able to avoid the path leading to the devalued end-box on the first trial after revaluation, even though they had never experienced the trajectory following the devaluation. Linear RL is also able to correctly update its plans using the DR computed in the learning phase (Fig $3 \mathrm{~b}-\mathrm{c}$ ). In particular, during the revaluation phase, the reward associated with one of the end-boxes changes but the structure of the environment remains the same: the revaluation corresponds to a change in $\mathbf{r}$ but not $\mathbf{M}$. Therefore, the agent is able to use the DR computed during the learning phase in the test phase and update its policy according to revalued reward function.

The SR is also capable of solving the latent learning task (and similar reward devaluation tasks with only a single step of actions widely used in neuroscience(Dickinson and Balleine, 2002)), because the SR, $\mathbf{S}^{\pi}$, even though learned under the original policy $\pi$, is good enough to compute usable new values from the new reward vector(Russek et al., 2017). However, there are many other, structurally similar revaluation tasks - in particular, those with several stages of choices - that defeat the SR. We considered a slightly different revaluation task, which Russek et al.(Momennejad et al., 2017; Russek et al., 2017) termed "policy revaluation" that has this property. Here human subjects were first trained to navigate a threestage sequential task leading to one of the three terminal states (Fig 3d(Momennejad et al., 2017)). The training phase was followed by a revaluation phase, in which participants experienced the terminal states with potentially new reward. In particular, a new large reward was introduced at a previously disfavored terminal state. In the final test, participants were often able to change their behavioral policy at the starting state of the task, even though they had never experienced the new terminal state contingent on their choices in the task(Momennejad et al., 2017).

Importantly, this is not possible for the SR without relearning or recomputing the successor matrix $\mathbf{S}^{\pi}$, because under the original training policy, the cached successor matrix does not predict visits to the previously low-valued state(Lehnert et al., 2017; Russek et al., 2017). That is, it computes values for the top-level state ( 1 in Fig $3 \mathrm{~d}$ ) under the assumption of outdated choices at the successor state (2), neglecting the fact that the new rewards, by occasioning a change in choice policy at 2 also imply a change in choice policy at 1 . This task then, directly probes the agent's ability to re-plan respecting the interdependence of optimal choices across states. Unlike the SR, linear RL can successfully solve this task using the DR that has 
been computed in the training phase, because the DR is independent of the decision policy in the learning phase (Fig 3e).

We finally considered a different class of replanning tasks, in which the transition structure of the environment changes, for example by placing a barrier onto the maze as to block the previously preferred path(Tolman, 1948). These tasks pose a challenge for both the SR and DR, since the environmental transition graph is cached inside both $\mathbf{S}^{\pi}$ and $\mathbf{M}$ (Momennejad et al., 2017; Russek et al., 2017), and these must thus be updated by relearning or recomputation in order to re-plan. However, people are again often able to solve this class of revaluations(Momennejad et al., 2017). We introduce an elaboration to linear RL to permit efficient solution of these tasks: in particular, we exploit matrix identities that allow us to efficiently update $\mathbf{M}$ in place to take account of local changes in the transition graph, then re-plan as before (see Methods). With these in place, the linear RL model can solve this task efficiently and computes the modified values and optimized policy using the old DR after updating it with simple operations (Fig 3h).

a
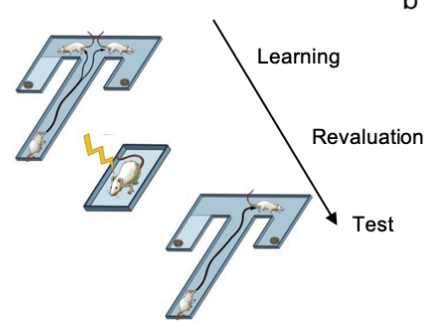

d

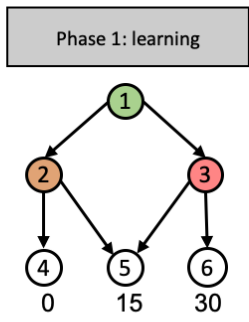

$\begin{array}{lll}45 & 5 & 6 \\ 40\end{array}$

f

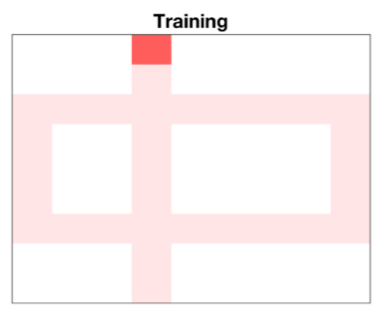

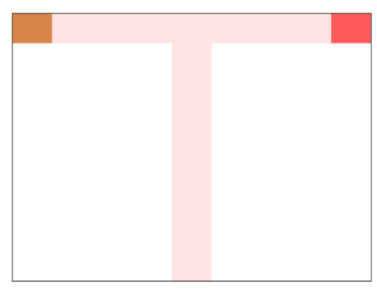

g

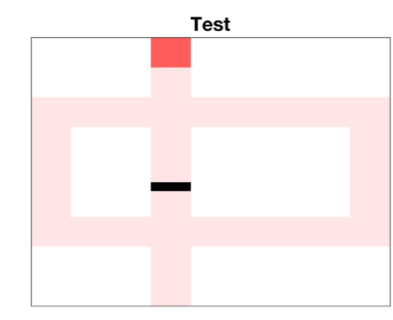

c

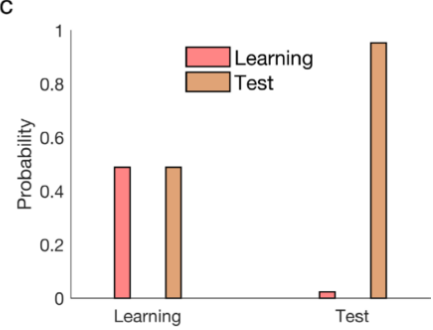

e

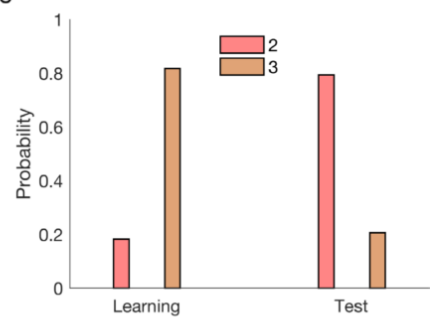

h

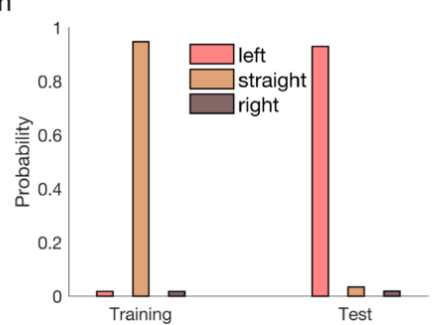

Fig 3. Linear RL can explain flexible replanning. a-c) Performance of linear RL on a version of Tolman's latent learning task (a). We simulated the model in a maze representing this task (b) and plotted the probability of choosing each end-box during the learning and test phases. The model correctly (c) reallocates choices away from the devalued option. d-e) Performance of linear RL in another reward revaluation task (Momennejad et al., 2017), termed policy revaluation (d). Choices from state 1: During the learning phase, the prefers to go to state 3 rather than state 2 . Revaluation of the bottom level state reverses this preference (e) similar to human subjects (Momennejad et al., 2017). f-h) Performance of the model in Tolman's detour task. The structure of the environment changes in this 
task due the barrier placed into the maze (g), which blocks the straight path. The model is able to compute the optimized policy using the old DR (following a single, inexpensive update to it) and correctly choose the left path in the test phase (h).

\section{Grid fields}

The linear RL model also highlights, and resolves, a central puzzle about the neural representation of cognitive maps or world models. It has long been argued that the brain represents a task's structure in order to support planning and flexible decision making(Tolman, 1948). This is straightforward for maximally local information: e.g., the one-step transition map $P\left(s_{t+1} \mid s_{t}, a_{t}\right)$ from Eq. 1, might plausibly be represented by connections between place fields in hippocampus, and combined with local-state reward mappings $r\left(s_{t}\right)$ that could be stored in hippocampal-stratial projections. But using this information for planning requires exhaustive evaluation, e.g. by replay(Mattar and Daw, 2018), and strongly suggesting a role for map-like representations of longer-scale relationships (aggregating multiple steps) to simplify planning(Botvinick et al., 2009; Sutton, 1995).

Indeed, grid cells in entorhinal cortex represent long-range (low-frequency) periodic relationships over space, and theoretical and experimental work has suggested that they play a key role in representation of the cognitive map and support navigation in both physical(Hafting et al., 2005) and abstract(Behrens et al., 2018; Constantinescu et al., 2016) state spaces. However, the specific computational role of these representations in flexible planning is still unclear. A key concept is that they represent a set of basis functions for quickly building up other functions over the state space, including future value predictions like $\bar{v}^{*}$ (Gustafson and Daw, 2011) and also future state occupancy predictions like the SR(Baram et al., 2018; Stachenfeld et al., 2017). By capturing longer range relationships over the map, such basis functions could facilitate estimating or learning these functions(Gustafson and Daw, 2011). In particular, the graph Laplacian (given by the eigenvectors of the on-policy, random walk transition matrix or, equivalently the eigenvectors of the SR for the random walk policy) generalizes Fourier analysis to an arbitrary state transition graph, and produces a set of periodic functions similar to grid fields(Mahadevan and Maggioni, 2007; Stachenfeld et al., 2017), including potentially useful low-frequency ones.

The puzzle with this framework is that, as mentioned repeatedly, the long-range transition map is not actually stable under changes in goals, since it depends on action choices ("max") at each step of Eq. 1: in effect, the spatial distribution of goals biases what would otherwise be a pure map of space, since those affect choice policy, which in turn affects experienced long-run location-location contingencies. Conversely, basis functions built on some fixed choice policy (like the SR for a particular $\pi$ ) are of limited utilty for transferring to new tasks(Lehnert et al., 2017; Russek et al., 2017). Accordingly, algorithms building on these ideas in computer science (such as "representation policy iteration,"(Mahadevan, 2012)), iteratively update basis functions to reflect changing policies and values as each new task is learned. It has been unclear how or whether representations like this can usefully support more one-shot task transfer, as in the experiments discussed in the previous section.

As shown in the previous section, linear RL resolves this problem, since the DR is similar to the SR but stably useful across different reward functions and resulting choice policies. In particular, the comparison 
between Eqs. 2 and 3 shows that the DR is a stable linear basis for the (approximate) optimal value function regardless of the reward function, but the SR is not. Accordingly, we suggest that grid cells encode an eigenvector basis for the DR, functions which are also periodic and have grid-like properties in 2D environments (Fig 4d). Empirically, because both the SR and DR represent relationships under the objective transition graph (e.g., barrier locations in space), both theories that grid fields should be affected by changes in the objective transition contingencies of the environment (e.g., barrier locations in space; though see the next section for another way to address this). This is indeed the case experimentally(Carpenter et al., 2015; Derdikman et al., 2009) (Fig. 4abc). However, the key experimental prediction is that grid fields based on the DR should be stable under changes in the choice policy, whereas the SR (and its eigenvectors) are strongly policy-dependent, so grid fields based on it should change to reflect the animal's tendency to follow particular trajectories(Stachenfeld et al., 2017). Experimental data strongly support the DR's prediction that grid fields are robust to behavioral changes; for instance, grid cells are affected by walls producing a "hairpin maze" but in rats trained to run an equivalent hairpin pattern without barriers(Derdikman et al., 2009) (Fig. 4ab); grid cells are also affected by the presence or absence of a set of walls the same shape as the animal's home cage, but whether or not it is the actual home cage (which strongly affects behavioral patterns) does not change the responses(SanguinettiScheck and Brecht, 2019) (Fig. 4c). Similar results have been reported in humans using functional neuroimaging(He and Brown, 2019). A second difference between the SR and the DR is that the DR (and its eigenvectors) include information about local costs along a path, so we predict that environmental features that make locomotion difficult, like rough terrain or hills, should modulate grid responses (see Discussion). 
a
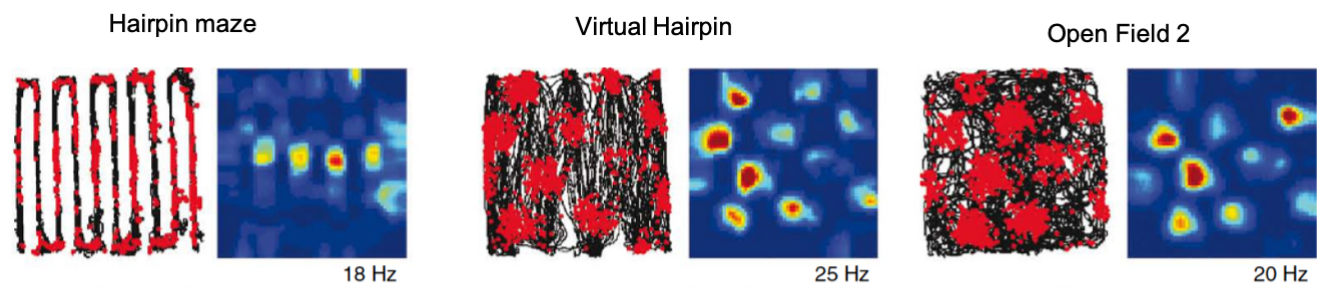

b

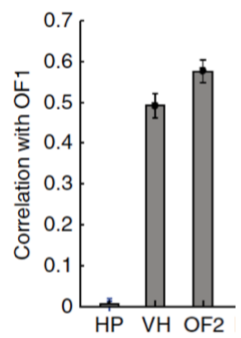

C
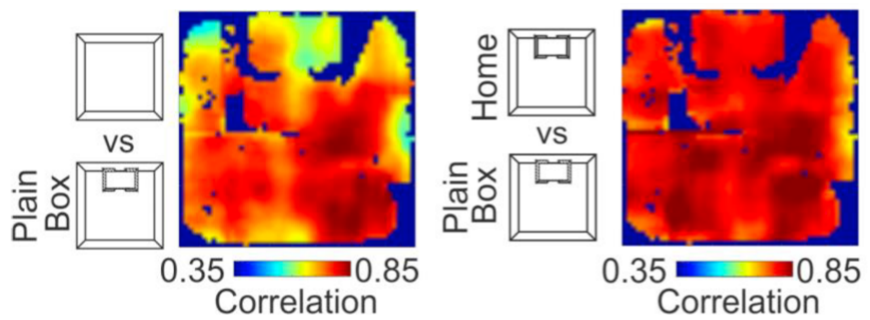

d
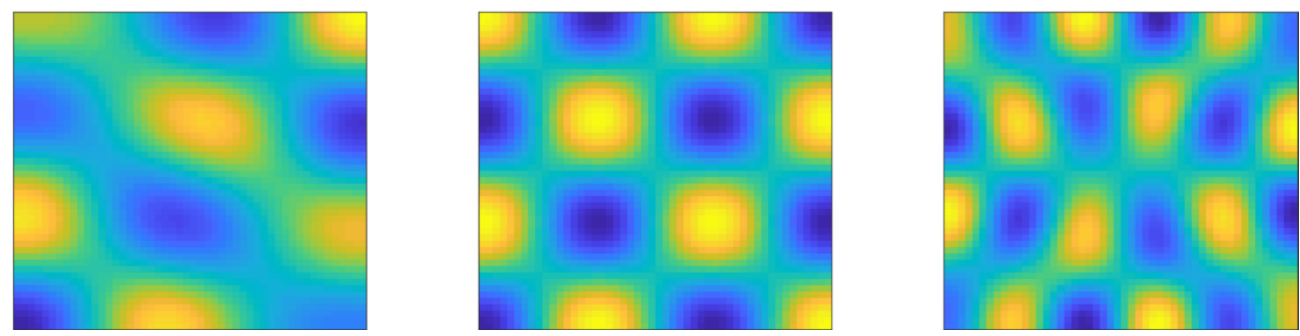

Fig 4. The DR as a model of grid fields. a-b) (adapted from Derdikman et al., 2009) Grid fields are sensitive to the geometry of the environment, but are stable with respect to behavior. Derdikman et al.(Derdikman et al., 2009) tested grid fields in a hairpin maze formed by actual barriers, and compared them to those recorded in a "virtual" hairpin maze, in which rats were trained to show hairpin-like behavior in an open field without constraining side walls. Grid fields in the virtual hairpin differ from those in the hairpin maze but are similar to the open field. b) This similarity is quantified by the correlation between grid fields in a baseline from an initial open field test (OF1) and those from the three tasks (HP: hairpin maze; VH: virtual hairpin; OF2: the second control open field). c) (Adapted from Sanguinetti-Scheck and Brecht(Sanguinetti-Scheck and Brecht, 2019)) Grid fields are sensitive to the presence of the home cage only insofar as it introduces new barriers in space, but not through the changes it produces in behavior. In particular, introducing a plain box (the same shape as the home cage) affects grid fields compared to the open field (left); but substuting the home cage for the box (right) does not further affect the grid code, although it changes behavior. d) Eigenvectors of the DR are independent from behavioral policies and periodic, similar to grid fields. Three example eigenvectors from a 50-by-50 maze are plotted.

\section{Border cells}

As we have already shown, one aspect of the environment that does require updating the DR if it changes is the transition structure of the environment, such as barriers. In simulating the Tolman detour task (Fig 
3c) we solved this problem using a matrix inversion identity, which rather than expensively recomputing the entire DR with respect to the new transition graph, expresses the new DR as the sum of the original DR plus a low-rank correction matrix reflecting, for each pair of states, the map change due to the barrier. This suggests a novel, componential way to build up spatial distance maps, such as the DR, by summing basis functions that correspond to generic components, like walls. In this case, grid cells could represent a low-rank (e.g. eigenvector) representation for a baseline map, and other cells could represent the contribution of additional environmental features. Here, we highlight the relevance and importance of this computational approach in the context of entorhinal border cells (Fig 5a). This is another principal family of neurons in the medial entorhinal cortex that fire exclusively when the animal is close to a salient border of the environment(Solstad et al., 2008), such as the wall; and are generic in the sense that they retain this tuning at least across changes in the environment's geometry. Assuming that the DR has been represented using a combination of features from a low-rank basis set, such as its eigenvectors, the columns of the matrix term for updating the DR show remarkable similarity to the border cells (Fig 5b). This brings the border cells and grid cells under a common understanding (both as basis functions for representing the map), and helps to express this map in terms of more componential features, like walls.

a

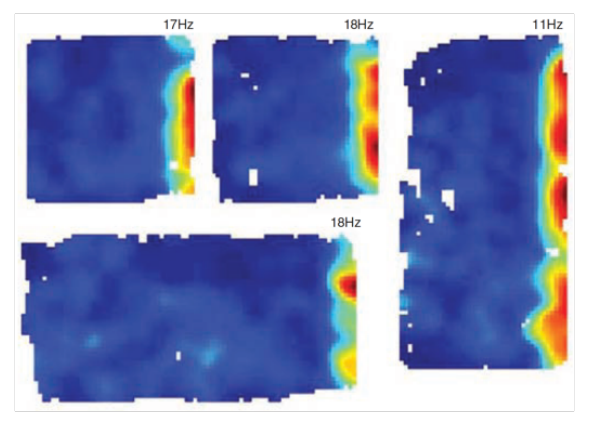

b
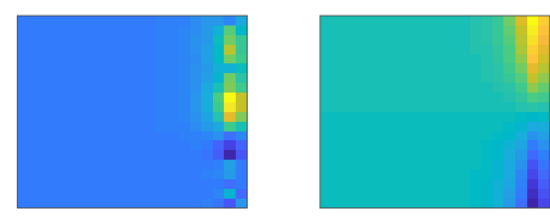
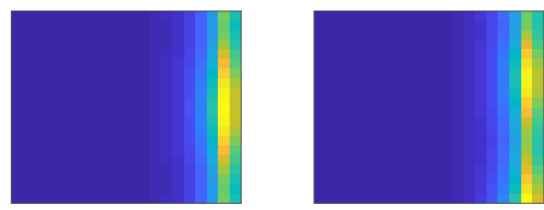

Fig 5. The model explains boundary cells. a) Adapted from Solstad et al.(Solstad et al., 2008), which shows rate maps for a representative border cell in different boxes. b) Columns of the matrix required to update the DR matrix to account for the wall resemble border cells. Four example columns from a 20-by-20 maze are plotted.

\section{Cognitive control}

We have stressed the usefulness of linear RL for enabling flexible behavior, but because of the inclusion of the default policy, the model also offers a natural framework for understanding biases and inflexibilities in behavior, and phenomena of cognitive control for overcoming them - as necessary consequences of 
the very same computational mechanisms that permit flexibility. The default policy represents soft, baseline assumptions about action preferences, which (on this view) are introduced because they help efficiently to solve the problem of forecasting the set of optimal future choices during planning. So far, we have simulated it as unbiased (uniform over successors), which works well; but in situations with stable, clear regularities in behavior, it can be an even better approximation to build these in via a nonuniform default. If the default policy is not uniform, it softly biases the model towards actions that are common under the default policy. This aspect of the model naturally captures biases in human behavior, such as Stroop effects and Pavlovian biases (next section), and suggests a novel rationale for them in terms of the default policy's role in facilitating efficient planning.

Cognitive control has been defined as the ability to direct behavior toward achieving internally maintained goals and away from responses that are in some sense more automatic but not helpful in achieving those goals(Botvinick and Cohen, 2014; Cohen et al., 1990). Two classic puzzles in this area are, first, why are some behaviors favored in this way; and second, why do people treat it as costly to overcome them(Kool et al., 2010; Shenhav et al., 2017; Westbrook et al., 2013)? For instance, is there some rivalrous resource or energetic cost that makes some behaviors feel more difficult or effortful than others(Kurzban et al., 2013; Shenhav et al., 2017)? Such "control costs" arise naturally in the current framework, since actions are penalized if they are more unlikely under the default policy. Such deviations from default are literally charged in the objective function, in units of reward: though for computational reasons of facilitating planning, rather than energetic ones like consuming a resource.

These control costs trade off in planning against the rewards for different actions, and lead (through the stochastic resulting policy) to biased patterns of errors. Fig $6 a, b$ plots the control cost as a function of the decision policy, showing that the cost is substantially larger for choosing the action that is less likely under the default policy. For instance, action A in this simulation could be the color-naming response in the classic Stroop task, in which participants must read the name of a color that it is printed in a differently colored ink. People are faster and make fewer errors in word reading compared to color naming, presumably because the former is a more common task. For the same reason, we would expect color naming to be less likely under the default policy, and incur a larger control cost to execute reliably (Fig $6 b)$. For any particular reward function (utility for correct and incorrect responses), this results in a larger chance of making errors for this action: a classic Stroop effect. Furthermore, since the optimal policy in the linear RL model balances the expected reward with the control cost, the model correctly predicts that these Stroop biases can be offset by increasing the rewards for correct performance(Botvinick and Braver, 2015) (Fig 6c). In other words, the prospect of reward can enhance performance even when the task is very difficult, as has been shown experimentally(Botvinick and Braver, 2015; Krebs et al., 2010). 

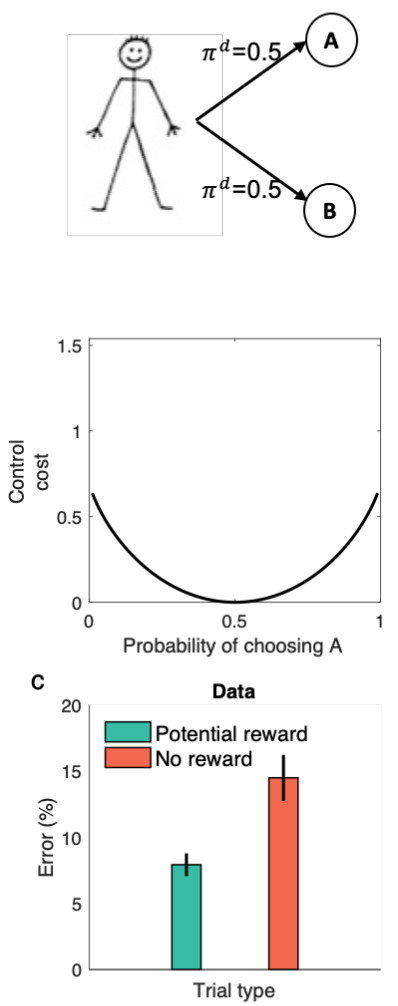

b
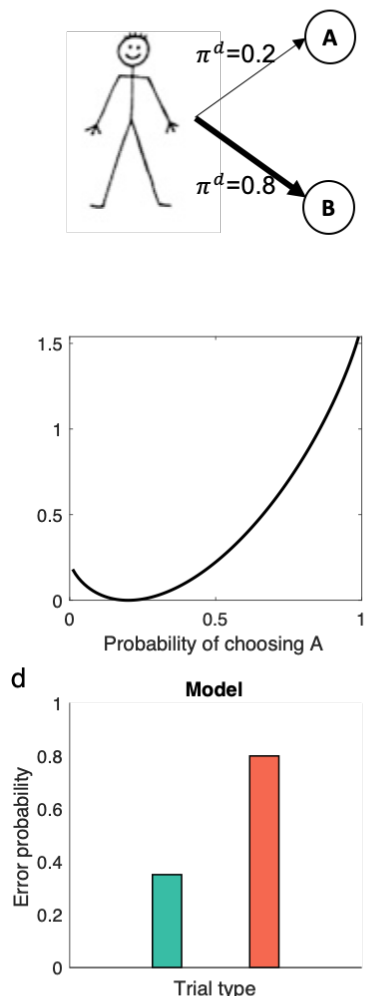

Fig 6. Linear RL captures prepotent actions and costs of cognitive control. a-b) The control cost is plotted as a function of the decision policy. For a uniform distribution (a) as the default policy, the control cost is a symmetric function of the decision policy. When the default policy is skewed toward a more likely response (b), the control cost is higher for reliably performing the action that is more unlikely under the default. c) People show classical Stroop effect in a color-naming Stroop task in which the name of colors are printed in the same or different color (Krebs, Boehler, \& Woldorff, 2010). These errors, however, are reduced in potential reward trials, in which correct response is associated with monetary reward. d) The linear RL model shows the same behavior, because the default probability is larger for the automatic response (i.e. word reading). Promising reward reduces this effect because the agent balances expected reward against the control cost to determine the optimized policy.

\section{Pavlovian-instrumental transfer}

A second example of response biases in the linear RL model arises in Pavlovian effects. In particular, PIT is a phenomenon by which previously learned Pavlovian stimulus-outcome relationships influence later instrumental decisions (action choices). Puzzlingly, this happens even though the Pavlovian cues are objectively irrelevant to the actions' outcomes(Corbit and Balleine, 2016; Dickinson and Balleine, 1994). PIT - in this case, associations between drug-associated cues and drugs triggering drug-seeking actions has been argued to play a key role in the development of addiction and cue-induced relapse(Everitt and Robbins, 2016). 
In a typical PIT task (Fig 7a), animals first learn that a neutral stimulus, such as a light, predicts some rewarding outcome in a Pavlovian phase. Later, in an instrumental phase, they learn to press a lever to get the same outcome. In the final testing phase, the presentation of the conditioned stimulus biases responding toward the action for the associated reward, even though the stimulus has never been presented during instrumental phase and the stimulus is objectively irrelevant as the action produces the outcome either way (Fig 7b). Existing RL models typically fail to explain this result, instead predicting that the presence of the stimulus should not influence behavior in the test phase, because actions predict the same outcome contingencies regardless of the stimulus.

Linear RL explains PIT as another example of biases arising from a learned default policy, because during the Pavlovian phase the agent learns that the reward outcome occurs more often in the presence of the conditioned stimulus, which is reflected in the default contingencies. Therefore, during the test phase, the presentation of a conditioned stimulus elicits a default policy biased toward the corresponding outcome occurring, which favors choosing the corresponding action (Fig 7c). Furthermore, this effect is carried by the sensory (state) aspects of the outcome, not its rewarding properties per se. In particular, since in the absence of reward, the decision policy is equal to the default policy, the theory predicts that PIT effects persist even in the absence of reward, which is consistent with experimental work showing that PIT biases survive even under reward devaluation (e.g. for food outcomes tested under satiety) (Fig $7 d-e)$. This finding that PIT effects reflect some sort of sensory cuing, and not reward or motivational properties of the stimulus per se, is central to the hypothesis that they underlie some phenomena in drug abuse such as cue-elicited relapse following extinction(Everitt and Robbins, 2016). 
a

\begin{tabular}{|c|c|}
\hline $\begin{array}{c}\text { Pavlovian } \\
\text { training }\end{array}$ & $\begin{array}{c}\text { Stimulus } 1 \Rightarrow \text { Outcome } 1 \\
\text { Stimulus } 2 \Rightarrow \text { Outcome 2 }\end{array}$ \\
\hline $\begin{array}{c}\text { Instrumental } \\
\text { training }\end{array}$ & $\begin{array}{c}\text { Response } 1 \Rightarrow \text { Outcome } 1 \\
\text { Response } 2 \Rightarrow \text { Outcome 2 }\end{array}$ \\
\hline PIT tests & $\begin{array}{c}\text { Stimulus } 1 \Rightarrow \text { Response } 1 \text { or } 2 ? \\
\text { Stimulus } 2 \Rightarrow \text { Response } 1 \text { or } 2 ?\end{array}$ \\
\hline
\end{tabular}
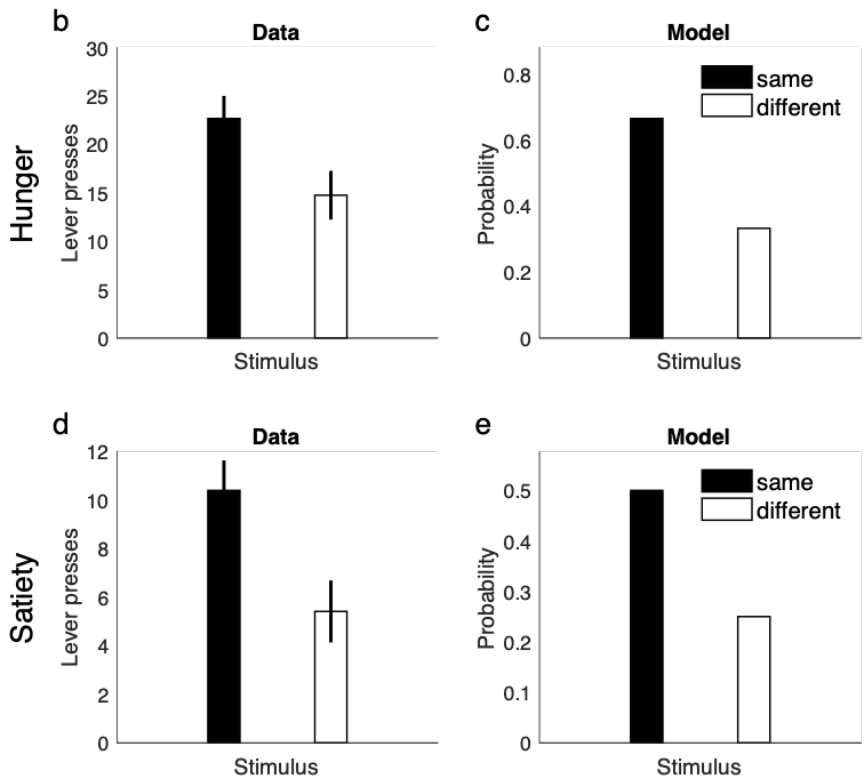

Fig 7. Linear RL explains Pavlovian-instrumental transfer. a) the task testing outcome-specific PIT consists of three phases: a Pavlovian training phase, an instrumental training phase and the PIT test. Outcomes 1 and 2 are both rewarding. During PIT test, both stimuli are presented in succession, and "same" responses denote the one whose associated outcome matches that associated with the presented stimulus, e.g. Response 1 chosen following presentation of Stimulus 1 . The other response is "different." b-c) Data from Corbit et al.(Corbit et al., 2007) when rats are hungry (b) and simulated behavior of the model (c). The model learns the default policy during the Pavlovian phase, which biases performance during the PIT test. d-e) Outcome-specific PIT persists even when rats are sated on both outcomes(Corbit et al., 2007) (d). The model shows the same behavior (e) because default state probabilities learned during Pavlovian training influence responses even in absence of reward. Mean and standard error are plotted in $\mathrm{b}$ and $\mathrm{c}$.

\section{Discussion}

A central question in decision neuroscience is how the brain can store cognitive maps or internal models of task contingencies and use them to make flexible choices, and more particularly how this can be done efficiently in a way that facilitates re-use of previous computations and leverages long-run, temporally abstract predictions without compromising flexibility. To help answer this question, we identify a core issue underlying many difficulties in planning, replanning, and reuse, which is the interdependence of optimal actions across states in a sequential decision task. To solve this problem, we import from control theory(Todorov, 2007, 2008) to neuroscience a novel computational model of decision making in the 
brain, called linear RL, which enables efficient (though approximate) global policy optimization by relying on soft relaxation away from default, stochastic policy expectations. This leverages the DR, a stored, longrun predictive map of state and cost expectancies under the default policy. The DR is closely related to the SR, and inherits many of the appealing features that have generated current excitement for it as a neuroscientific model(Gershman, 2018; Momennejad et al., 2017; Russek et al., 2017; Stachenfeld et al., 2017). However, linear RL corrects serious problems that hobble the practical applicability of the SR. The DR, unlike the SR, exerts only a weak bias toward the default policy, and so delivers on the promise of a stable cognitive map(Tolman, 1948) that can reuse substantial computation to transfer learning across contexts without sacrificing flexibility. This allows the model to explain animals' ability to solve reward and policy revaluation problems that otherwise would require exhaustive, biologically unrealistic modelbased search. For the same reason, the model also helps to deliver on the idea that grid cells in entorhinal cortex could provide a broadly useful neural substrate for such a temporally abstract map. And the model's remaining inflexibilities - in general, soft, stochastic biases rather than hard failures - connect naturally with phenomena of cognitive control and Pavlovian biases and provide a strong theoretical framework for understanding the role of many such biases in both healthy and disordered choice.

The basic planning operation in linear RL is matrix-vector multiplication, which is easily implemented in a single neural network layer. The theory offers new insights into the systems-level organization of this computation. In particular, the model realizes the promise of a representation that factors a map representing the structure of environment, separate from an enumeration of the current value of the goals in the environment. This facilitates transfer by allowing update of either of these representations while reusing the other. Previous models, like the SR, nominally exhibit this separation, but the hard policy dependence of the SR's state expectancies means that goal information, in practice, inseparably infects the map and interferes with flexible transfer(Lehnert et al., 2017; Russek et al., 2017).

In fact, in order to facilitate efficient planning, the linear RL model actually factors the map into three rather than two pieces, distinguishing between terminal states (representing goals), and nonterminal states (those that may be traversed on the way to goals); and dividing the map into one matrix encoding long-run interconnectivity between nonterminal states (the $\mathrm{DR}, \mathbf{M}$ ) and a second matrix representing onestep connections from nonterminal states to goals $(\mathbf{P})$. This is a necessary restriction, in that only for this type of finite decision problem are the optimal values linearly computable. However, this classification is not inflexible, because we also introduce novel techniques (based on matrix inversion lemmas) that allow dynamically changing which states are classed as goals. This allows the model (for example) to plan the best route to any arbitrary location in a maze (Fig $2 \mathrm{~d}$ ). Representing goals as terminal states also means that the model does not directly solve problems that require figuring out how best to visit multiple goals in sequence. However, this restriction has little effect in practice because these can either be treated as a series of episodes, re-starting at each goal, or by including multiple goals within the nonterminal states, since the model does optimize aggregate rewards over trajectories through nonterminal states as well.

This last point raises several interesting directions for future work. First, although there is evidence that humans choose their goal and plan towards that goal(Cushman and Morris, 2015), there is some empirically underconstrained theoretical flexibility in specifying how a task's state space should be 
partitioned into terminal and nonterminal states. For the simulations here, we have tentatively adopted the principle that all discrete, punctate outcomes (like food or shock) are represented as terminal goal states with corresponding value in $\mathbf{r}$, and the rest of the (nonterminal) states contain only costs, constant everywhere, meant to capture the cost of locomotion. But, in general, state-dependent costs (or indeed rewards) can be included for nonterminal states as well. These in effect modulate the "distance" between states represented within the DR (see Methods). Nevertheless, this leads to the testable prediction that to whatever extent state-specific rewards or costs are accounted for within nonterminal states, they should affect hypothetical neural representations of the DR, such as grid cells. For instance, unlike for the $\mathrm{SR}$, the DR predicts that by increasing locomotion cost, hills or rough terrain should increase "distance" as measured in the grid map. This aspect of the DR may be relevant for explaining recent evidence that grid cells have some subtle sensitivities to reward(Boccara et al., 2019; Butler et al., 2019) which cannot be explained, as the SR-eigenvector account would predict, as secondary to changes in behavioral policy (e.g., not due to occupancy around rewarding locations(Butler et al., 2019), nor variations in trajectories or speed(Boccara et al., 2019)).

Linear RL requires one other formal restriction on tasks, compared to standard Markov decision processes as often assumed by other RL theories in theoretical neuroscience. This is that the task is deterministically controllable. This is a good fit for many important sequential tasks, such as spatial navigation (I can reliably get from location A to location B by taking a step forward) and instrumental lever-pressing, but does not directly or exactly map to tasks that include irreducibly stochastic state transitions, such as two-step noisy Markov decision tasks that we and others have used to study model-based planning(Daw et al., 2011). Such tasks can also be addressed via a further step of approximation(Todorov, 2009), but it remains for future work to explore how far this can be pushed.

We have stressed how the DR can be used for planning, and also how it embodies substantial, reusable computation (specifically, predictions of long-run future state occupancy and cost-to-go), relative to simpler, easy-to-learn map representations like the one-step state adjacency model $P\left(s_{t+1} \mid s_{t}\right)$. We have not, so far, discussed how the DR can itself be learned or computed. There are several possibilities: two inherited from previous work on the SR(Russek et al., 2017) and one newly introduced here. First, like the $\mathrm{SR}$, the DR can be learned gradually by actual or replay-based sampling of the environment, using a temporal difference rule(Dayan, 1993; Russek et al., 2017). Second, again like the SR, the DR can be constructed from the one-step transition matrix and costs (which can themselves be learned directly by Hebbian learning) by a matrix inversion, or equivalently a sum over a series of powers of a matrix. The latter form motivates attractor methods for computing the inverse iteratively by a simple recurrent network(Jang et al., 1988; Russek et al., 2017; Sutton and Pinette, 1985).

A third possibility for learning the DR follows from the novel method we introduce for using matrix inversion identities to efficiently update the DR in place to add additional goals, barriers, or shortcuts (see Methods). This works by expressing the inverse matrix in terms of the inverses of simpler component matrices (one of which is the pre-update DR), rather than for instance by updating the transition matrix and then, expensively, re-inverting the whole thing. For instance, we used this to solve tasks, such as Tolman's detour task, in which the transition structure of the environment changes. It could also be used, 
state by state or barrier by barrier, as a learning rule for building up the DR from scratch. Suggestively, this insight that the Woodbury matrix inversion identity can be used to decompose a DR map (an inverse matrix) into the sum of component maps, each associated with different sub-graphs of the transition space, offers a very promising direction for a direct neural implementation for representing and constructing maps componentially: via summing basis functions. This idea dovetails with - and may help to formalize and extend - the emerging idea that maps in the brain are built up by composing basis functions, such as those putatively represented in the grid cells(Baram et al., 2018; Behrens et al., 2018; Dordek et al., 2016; Stachenfeld et al., 2017; Whittington et al., 2019). Here, we showed that the term required to update the DR when encountering a wall remarkably resembles entorhinal border cells(Solstad et al., 2008). Therefore, our theory unifies the functional roles of entorhinal grid and border cells in planning and navigation, both as basis functions for building up the map. It remains for future work to explore the extent that this technique can be used to account for other aspects of neural coding in the entorhinal cortex. With respect to the grid cells, we also note that just as for the graph Laplacian and SR(Gustafson and Daw, 2011; Stachenfeld et al., 2017), the eigenvectors of the DR capture the periodicity and multiscale aspect of the grid cell code, but only a subset of them exhibit hexagonal symmetry. Additional constraints, such as nonnegativity(Dordek et al., 2016) are likely required for a more detailed model.

Our model is based on the notion of the default policy, which is a map of expected state-to-state transition probability regardless of the current goals. Unlike previous RL models, such as the SR, linear RL does not entirely rely on the default policy and instead optimizes the decision policy around the default policy. This means that the final optimized policy is between the exact, deterministic optimized policy, and the default. The degree of this bias is controlled by a free parameter that scales the control costs relative to rewards and corresponds to the temperature in the softmax approximation to the optimization. In the limit of zero, or respectively infinite, control cost scaling, the approximation to the optimum becomes exact, or the default policy dominates completely. How should this parameter be set, and why not always take it near zero to improve the fidelity of the approximation? Linear RL works by multiplying small numbers (future occupancies) times large numbers (exponentiated, scaled rewards) to approximate the maximum expected value; just as with numerical precision in computers, there are issues of bandwidth (e.g. maximum spike rate and quantization) and gain control for making this work effectively across different decision situations in the brain. This suggests fruitful connections (for future work) with gain control and normalization(Louie et al., 2011), and rational models for choice using noisy representations(Gershman and Wilson, 2010; Woodford, 2012). The same tradeoff can also be understood from a Bayesian planning as inference perspective(Botvinick and Toussaint, 2012), in which the default policy plays the role of prior over policy space and rewards play the role of the likelihood function. In this case, the decision policy is the posterior that optimally combines them(Todorov, 2008). Then, how much the decision policy should be influenced by the default depends on how informative a prior it is (e.g. how reliable or uncertain it has been previously). This also suggests another distinct perspective on the default policy's role, in the model, in producing prepotent biases that can be overcome by cognitive control(Botvinick and Cohen, 2014; Shenhav et al., 2017). On this view, it serves to regularize 
behavior toward policies that have worked reliably in the past; and deviations from this baseline are costly.

Indeed, our framework leaves open not just how strongly the default policy is emphasized, but also how it is learned or chosen. In general, while the model provides a good approximation to the true optimal values independent of which default policy is used (so long as its cost is scaled appropriately relative to the rewards), we can also ask the converse question - which default policy should be chosen to allow for the best approximation and thereby obtain the most (actual) reward? The answer is of course, that the cost term (measuring the divergence between true and approximate $\mathbf{v}^{*}$ ) is minimized whenever the future $\pi^{*}$ is equal to the default $\pi^{d}$. Any algorithm for learning policies might be appropriate, then, for finding a $\pi^{d}$ that is likely to be near-optimal in the future, including in particular previous habit learning models, including model-free actor-critic learning(Barto, 1995) or even non-reward-driven memorization of previous policies(Miller et al., 2019). A related idea has also been recently proposed in the context of a more explicitly hierarchical model of policy learning: that a default policy (and control-like charges for deviation form it) can be useful in the context of multitask learning to extract useful, reusable policies(Kool and Botvinick, 2018; Teh et al., 2017). Separately, an analogous principle of identification of task structure that generalizes across tasks in a hierarchical generative model has also been proposed as a model of grid and place cell responses(Behrens et al., 2018; Whittington et al., 2019). Future work remains to understand the relationship between the considerations in both of these models - which involve identifying shared structure - and ours, which are motivated instead more by efficiently reusing computation.

The role of the default policy, finally, points at how the linear RL framework provides a richer, more nuanced view of habits and pathologies of decision making than previous computational theories. Although a learned default policy biases behavior, and may modulate accuracy or speed of performance, it trades off against rewards in the optimization. This give and take stands in contrast to much previous work, especially in computational psychiatry, which has often assumed a binary model of evaluation: either flexible values are computed (model-based, goal-directed) or they are not (model-free, habits). The latter, acting rather than thinking, has been taken as a model of both healthy and unhealthy habits, and especially of compulsive symptoms such as in drug abuse(Everitt and Robbins, 2016) and obsessive compulsive disorder(Gillan et al., 2016). Although such outright stimulus-response behaviors may exist, the present framework allows for a much broader range of biases and tendencies, and may help to understand a greater range of symptomatology, such as excessive avoidance in anxiety(Zorowitz et al., 2019), craving and cue-induced relapse in drug abuse, and the ability to effortfully suppress compulsive behaviors across many disorders. Finally, and relatedly, the possibility of a dynamic and situationdependent default policy also offers a way to capture some aspects of emotion that have been resistant to RL modeling. In particular, one important aspect of emotion is its ability to elicit a pattern of congruent response tendencies, such as a greater tendency toward aggression when angry. Complementing recent work suggesting these might arise due to a hard bias on planning (via pruning context-inappropriate actions)(Huys and Renz, 2017), the default policy offers a clear and normative lever for influencing behavior on the basis of emotional (and other) context. 


\section{Methods}

\section{Model description}

In this work, we focus on Markov decision processes with two conditions. First, we assume that there is one or a set of terminal states, $s_{T}$; Second, we only consider deterministic environments, such as mazes, in which there is a one-to-one map between actions and successor states.

The linear RL model is then based on a modification to the value function for this setting(Todorov, 2007, 2009), in which the agent controls the probabilistic distribution over successor states (i.e., actions) and pays an additional control cost quantified as the dissimilarity (in the form of KL divergence) between the controlled dynamics (i.e. decision policy), $\pi\left(. \mid s_{t}\right)$ and a default dynamics, $\pi^{d}\left(. \mid s_{t}\right)$. In particular, the objective of this MDP is to optimize a "gain" function, $g\left(s_{t}\right)$, defined as

$$
g\left(s_{t}\right)=r\left(s_{t}\right)-\lambda \mathrm{KL}\left(\pi \| \pi^{d}\right)
$$

where $\lambda>0$ is a constant and $\operatorname{KL}\left(\pi \| \pi^{d}\right)$ is the $\mathrm{KL}$ divergence between the two probability distributions; it is only zero if the two distributions are the same, i.e. $\pi=\pi^{d}$ and otherwise is positive. We also require that $\pi=0$ if $\pi^{d}=0$. Note that in the limit of zero, or respectively infinite, $\lambda$, the gain converges to pure reward (i.e. a standard MDP), or pure cost. Here, $\lambda$ scales the relative strength of control costs in units of reward (and is equivalent to rescaling the units of reward while holding the cost fixed).

It is easy then to show that the optimal value function for this new problem, $\mathbf{v}^{*}$, is analytically solvable(Todorov, 2007, 2009) (see formal derivation below). We first define the one-step state transition matrix $\mathbf{T}$, whose $(i, j)$ element is equal to the probability of transitioning from state $i$ to state $j$ under the default policy (i.e. probability of the action under the default policy that makes $i \rightarrow j$ transition). This contains subblocks, $\mathbf{T}_{N N}$, the transition probability between nonterminal states, and $\mathbf{T}_{N T}=\mathbf{P}$, the transition probabilities from terminal to nonterminal states. Then:

$$
\exp \left(\mathbf{v}^{*} / \lambda\right)=\mathbf{M P} \exp (\mathbf{r} / \lambda)
$$

where $\mathbf{v}^{*}$ is the vector of optimal values at nonterminal states, $\mathbf{r}$ is the vector of rewards at terminal states, and $\mathbf{M}$ is a matrix defined below. Note that equation (3) is the case of this equation for $\lambda=1$.

The DR matrix $\mathbf{M}$ is defined as:

$$
\mathbf{M}=\left(\operatorname{diag}\left(\exp \left(-\mathbf{r}_{N} / \lambda\right)\right)-\mathbf{T}_{N N}\right)^{-1},
$$

where $\mathbf{r}_{N}$ is the vector of rewards at nonterminal states (which we take as a uniform cost of -1 in most of our simulations).

For flexibility in updating which states are viewed as goal states, it is helpful to define a second, more general version of the DR matrix, D, defined over all states (not just nonterminal states) as:

$$
\mathbf{D}=\left(\operatorname{diag}\left(\exp \left(-\mathbf{r}_{A} / \lambda\right)\right)-\mathbf{T}\right)^{-1},
$$

where $\mathbf{r}_{A}$ is the reward vector across all states. Note that since matrix $\mathbf{M}$ can be easily computed from $\mathbf{D}$ (in particular, $\mathbf{M}$ is a subblock of $\mathbf{D}$ corresponding to the nonterminal states only), we refer to both of them 
as the DR unless specified otherwise. Also note that for defining D, we assumed, without loss of generality (since this assumption does not affect $\mathbf{M}$ ), that reward at terminal states are not 0 .

This solution for $\mathbf{v}^{*}$ further implies that the policy takes the form of a weighted softmax, where the weights are given by the default policy

$$
\pi\left(a \mid s_{t}\right)=\frac{\pi^{d}\left(a \mid s_{t}\right) \exp \left(v^{*}\left(s_{a}\right) / \lambda\right)}{\sum_{a^{\prime}} \pi^{d}\left(a^{\prime} \mid s_{t}\right) \exp \left(v^{*}\left(s_{a^{\prime}}\right) / \lambda\right)}
$$

where $s_{a}$ is the successor state associated with action $a$. Thus, for a uniform default policy, the optimal policy is simply given by the softmax over optimal values with the temperature parameter $\lambda$. Note also that in the limit of $\lambda=0$, the problem becomes the classical MDP (because $g\left(s_{t}\right)=r\left(s_{t}\right)$ in equation (4)) and the decision policy in equation (6) also reflects the optimum policy (i.e. greedy) exactly. In the limit of infinite $\lambda$, the influence of the rewards vanishes and the decision policy converges to the default policy.

\section{Planning toward a new goal and transfer revaluation}

Consider an environment with $\mathbf{T}_{\mathbf{0}}$ and $\mathbf{D}_{\mathbf{0}}$ as the transition matrix under the default policy and the associated DR, respectively. Now suppose that the agent's goal is to plan toward state $j$ (or equivalently computing the distance between any state and $j$ ), i.e., we wish to add $j$ to the set of terminal states. Here, we aim to develop an efficient method to plan towards $j$ by using the cached $\mathbf{D}_{\mathbf{0}}$, without re-inverting the matrix.

If we define $\mathbf{L}_{\mathbf{0}}=\operatorname{diag}\left(\exp \left(-\mathbf{r}_{A} / \lambda\right)\right)-\mathbf{T}_{0}$ and $\mathbf{L}=\operatorname{diag}\left(\exp \left(-\mathbf{r}_{A} / \lambda\right)\right)-\mathbf{T}$, then $\mathbf{L}$ and $\mathbf{L}_{\mathbf{0}}$ are only different in their $j$ th row (because $\mathbf{T}$ and $\mathbf{T}_{\mathbf{0}}$ are only different in their $j$ th row). We define $\mathbf{d}$, a row-vector corresponding to the difference in $j$ th row of the two matrices:

$$
\mathbf{d}=\mathbf{L}(j,:)-\mathbf{L}_{0}(j,:) \text {, }
$$

and therefore, we can write:

$$
\mathbf{L}=\mathbf{L}_{\mathbf{0}}+\mathbf{d e} \text {, }
$$

where $\mathbf{e}$ is a binary column-vector that is one only on $j$ th element. Using the Woodbury matrix identity, $\mathbf{L}^{-1}$ is given by

$$
\mathbf{L}^{-1}=\mathbf{L}_{\mathbf{0}}^{-1}-\frac{1}{1+\mathbf{d L}_{\mathbf{0}}^{-1} \mathbf{e}} \mathbf{L}_{\mathbf{0}}^{-1} \mathbf{e d \mathbf { L } _ { \mathbf { 0 } } ^ { - 1 }}
$$

in which we exploited the fact that $\mathbf{d}$ and $\mathbf{e}$ are row- and column- vectors, respectively, and therefore $\mathbf{d L}_{\mathbf{0}}^{-1} \mathbf{e}$ is a scalar. Since $\mathbf{D}_{\mathbf{0}}=\mathbf{L}_{\mathbf{0}}^{-1}$ and $\mathbf{D}=\mathbf{L}^{-1}$, we obtain

$$
\mathbf{D}=\mathbf{D}_{\mathbf{0}}-\frac{1}{1+\mathbf{d m}_{\mathbf{0}}} \mathbf{m}_{\mathbf{0}} \mathbf{d D}_{\mathbf{0}}
$$

where $\mathbf{m}_{\mathbf{0}}$ is the $j$ th column of $\mathbf{D}_{\mathbf{0}}$.

The above equation represents an efficient, low-rank update to the DR itself. However, for the purpose of this single planning problem (e.g. if, we do not intend further modifications to the matrix later), we may also further simplify the computation by focusing only on the product $\mathbf{z}=\mathbf{M P}$, which is what is needed 
for planning using equation (5) in the new environment. We find $\mathbf{z}$ in terms of an intermediate vector $\hat{\mathbf{z}}=$ $\mathbf{D} \widehat{\mathbf{P}}$, where $\widehat{\mathbf{P}}$ is a subblock of $\mathbf{T}$ from all states to terminal states, in which all elements of rows corresponding to terminal states are set to 0 . Therefore, $\hat{\mathbf{z}}$ is given by

$$
\hat{\mathbf{z}}=\mathbf{z}_{\mathbf{0}}-\frac{1}{1+\mathbf{d m}_{\mathbf{0}}} \mathbf{m}_{\mathbf{0}} \mathbf{d \mathbf { z } _ { 0 }}
$$

where

$$
\mathbf{z}_{\mathbf{0}}=\mathbf{D}_{\mathbf{0}} \widehat{\mathbf{P}}
$$

Finally, $\mathbf{z}$ is given by the submatrix of $\hat{\mathbf{z}}$ corresponding to nonterminal rows.

It is important to note that since $\mathbf{d}$ and $\widehat{\mathbf{P}}$ are very sparse, computations in equations (8-9) are local. In fact, $\mathbf{d}$ is only nonzero on elements associated with immediate state of $j$ (and $j$ th element). If we assume that there is only one terminal state (i.e. $j$ ), then $\widehat{\mathbf{P}}$ is a vector that is nonzero on elements associated with immediate state of $j$.

The same technique can be used to update the DR or re-plan in transfer revaluation problems, such as localized changes in $\mathbf{T}_{N N}$ or $\mathbf{P}$. For example, if transition from state $j$ to $i$ has been blocked, new values for $\mathbf{D}$ and $\mathbf{z}$ can be computed efficiently using equations (7) and (8), respectively. Similarly, $\mathbf{D}$ and $\mathbf{z}$ can be computed efficiently using those equations if the reward value for the nonterminal state changes. Finally, it is also possible to learn the DR matrix, transition by transition, by iteratively computing $\mathbf{D}$ for each update using $\mathbf{D}_{0}$ in equation (7).

\section{Border cells}

We employed a similar approach to account for border cells. Suppose that a wall has been inserted into the environment, which changes the transition matrix $\mathbf{T}_{0}$ to $\mathbf{T}$. Suppose $\mathbf{L}_{\mathbf{0}}=\operatorname{diag}\left(\exp \left(-\mathbf{r}_{A} / \lambda\right)\right)-\mathbf{T}_{0}$ and $\mathbf{L}=\operatorname{diag}\left(\exp \left(-\mathbf{r}_{A} / \lambda\right)\right)-\mathbf{T}$. We define matrix $\boldsymbol{\Delta}$ using rows of $\mathbf{L}_{\mathbf{0}}$ and $\mathbf{L}$ corresponding to $J$ :

$$
\Delta=\mathbf{L}_{J}-\mathbf{L}_{0 J}
$$

where $J$ denotes those states that their transition has been changed, $\mathbf{L}_{J}$ and $\mathbf{L}_{0 J}$, are, respectively, submatrices associated with rows of $\mathbf{L}$ and $\mathbf{L}_{\mathbf{0}}$ corresponding to $J$. Using the Woodbury matrix identity (similar to equation (7)), the DR associated with the new environment is given by

$$
\mathbf{D}=\mathbf{D}_{\mathbf{0}}-\mathbf{B},
$$

where

$$
\mathbf{B}=\mathbf{D}_{\mathbf{0} J}\left(\mathbf{I}+\Delta \mathbf{D}_{\mathbf{0} J}\right)^{-1} \Delta \mathbf{D}_{\mathbf{0}}
$$

in which matrix $\mathbf{D}_{\mathbf{0} J}$ is the submatrix associated with columns of $\mathbf{D}_{\mathbf{0}}$ corresponding to $J$, and $\mathbf{I}$ is the identity matrix. Note that although this model requires inverting of a matrix, this computation is substantially easier than inverting matrix $\mathbf{L}$, because this matrix is low-dimensional. For simulating the border cells in Fig 5, we replaced matrix $\mathbf{D}_{\mathbf{0}}$ by its eigenvectors. Thus, if $\mathbf{u}$ is an eigenvector of $\mathbf{D}_{\mathbf{0}}$, the corresponding column in $\mathbf{B}, \mathbf{b}(\mathbf{u})$ is given by 


$$
\mathbf{b}(\mathbf{u})=\mathbf{D}_{\mathbf{0} J}\left(\mathbf{I}+\Delta \mathbf{D}_{\mathbf{0} J}\right)^{-1} \Delta \mathbf{u}
$$

\section{Simulation details}

We assumed a uniform default policy in all analyses presented in Figure 1-5. In Fig 1, the cost for all states were randomly generated in the range of 0 to 10 and analysis was repeated 100 times. In Fig 2 b-c, a 50x50 maze environment was considered. In Fig $2 \mathrm{~d}$-e, a 10x10 maze was considered with 20 blocked states. The DR was computed in this environment with no terminal state, in which the cost for all states was 1 . We used equation (8) to compute the shortest path using linear RL. The optimal path between every two states was computed by classic value iteration algorithm. In Fig 3b-c, the reward of all states was -1 , except the terminal states, which was +5 . In the revaluation phase, the reward of the left terminal state was set to -5 . In Fig 3d, the reward of states 1,2 and 3 is 0 . In Fig 3e, reward at all states is -1 , except for the terminal state, which is +5 . In Fig $4 d$, a $50 \times 50$ maze was considered, the cost for all states was assumed to be 0.1 . In this figure, $15^{\text {th }}, 20^{\text {th }}, 32^{\text {th }}$ eigenvectors of the DR have been plotted. In Fig $5 \mathrm{~b}$, a $20 \times 20$ maze was considered and the cost for all states was assumed to be 0.1 . In this figure, $1^{\text {th }}, 6^{\text {th }}, 11^{\text {th }}, 12^{\text {th }}$ eigenvectors of the DR have been considered.

The default policy in Figs 6-7 was not uniform. In Fig $6 c$, the default probability for the control-demanding action assumed to be 0.2 and reward was assumed to be +2 . For simulating PIT in Fig 7, we followed experimental design of Corbit et al.(Corbit et al., 2007) and assumed that the environment contains 4 states, in which state 1 was the choice state, states 2,3 , and 4 were associated with outcomes 1,2 and 3, respectively. In Fig 7c, the reward of outcome 1-3 was +5 . In Fig 7e, the reward of all states was assumed to be 0 . It was also assumed that during the Pavlovian training, the default probability for Stimulus $1 \rightarrow$ Outcome 1 and for Stimulus $2 \rightarrow$ Outcome 2 changes from 0.33 (i.e. uniform) to 0.5.

The only parameter of linear RL is $\lambda$, which was always assumed to be 1 , except for simulating the results presented in Fig $3 e$, where we set $\lambda=10$ to avoid overflow of the exponential due to large reward values.

\section{Formal derivation}

For completeness, we present derivation of equations (5-6) based on Todorov(Todorov, 2007, 2009). By substituting the gain defined in equation (4) into the Bellman equation (1), we obtain:

$$
v\left(s_{t}\right)=r\left(s_{t}\right)+\max _{\pi}\left\{-\lambda E_{a \sim \pi\left(a \mid s_{t}\right)}\left[\log \frac{\pi\left(a \mid s_{t}\right)}{\pi^{d}\left(a \mid s_{t}\right) \exp \left(v\left(s_{a}\right) / \lambda\right)}\right]\right\},
$$

where $s_{a}$ denotes the corresponding state (among the set of successor states of $s_{t}$ ) to action $a$.

Note that the expectation in the Bellman equation is under the dynamics, which we have replaced it with the policy because they are equivalent here. The expression being optimized in this equation is akin to a $\mathrm{KL}$ divergence, except that the denominator in the argument of the log function is not normalized. Therefore, we define the normalization term $c$ :

$$
c=\sum_{a} \pi^{d}\left(a \mid s_{t}\right) e^{v\left(s_{a}\right) / \lambda}
$$


Note that $c$ is independent of the distribution being optimized $\pi$. By multiplying and dividing the denominator of the $\log$ by $c$, we obtain:

$$
v\left(s_{t}\right)=r\left(s_{t}\right)+\lambda \log c+\max _{\pi}\left\{-\lambda \operatorname{KL}\left(\pi\left(a \mid s_{t}\right) \| \pi^{d}\left(a \mid s_{t}\right) e^{v\left(s_{a}\right) / \lambda} / c\right)\right\},
$$

where the maximum value of negative $\mathrm{KL}$ divergence is zero, which occurs only if the two distributions are equal, giving rise to equation (6):

$$
\pi\left(a \mid s_{t}\right)=\pi^{d}\left(a \mid s_{t}\right) e^{v\left(s_{a}\right) / \lambda} / c .
$$

Furthermore, since the KL divergence is zero, optimal values satisfy:

$$
v^{*}\left(s_{t}\right)=r\left(s_{t}\right)+\lambda \log c .
$$

Across all states, this gives rise to a system of linear equations in the exponential space. Since at terminal states, $v\left(s_{T}\right)=r\left(s_{T}\right)$, this system can be solved analytically, which can be written in the matrix equation 5.

\section{Acknowledgement}

We thank Tim Behrens and Jon Cohen for helpful discussions. This work was supported by grants IIS1822571 from the National Science Foundation, part of the CRNCS program, and 61454 from the John Templeton Foundation. 


\section{References}

Baram, A.B., Muller, T.H., Whittington, J.C.R., and Behrens, T.E.J. (2018). Intuitive planning: global navigation through cognitive maps based on grid-like codes. BioRxiv 421461.

Barto, A.G. (1995). Adaptive critic and the basal ganglia. In Models of Information Processing in the Basal Ganglia, J.C. Houk, J.L. Davis, and D.G. Beiser, eds. (Cambridge: MIT Press), pp. 215-232.

Behrens, T.E.J., Muller, T.H., Whittington, J.C.R., Mark, S., Baram, A.B., Stachenfeld, K.L., and Kurth-Nelson, Z. (2018). What Is a Cognitive Map? Organizing Knowledge for Flexible Behavior. Neuron 100, 490-509. Bellman, R.E. (1957). Dynamic Programming (Princeton, NJ: Princeton University Press).

Boccara, C.N., Nardin, M., Stella, F., O'Neill, J., and Csicsvari, J. (2019). The entorhinal cognitive map is attracted to goals. Science 363, 1443-1447.

Botvinick, M., and Braver, T. (2015). Motivation and cognitive control: from behavior to neural mechanism. Annu. Rev. Psychol. 66, 83-113.

Botvinick, M., and Toussaint, M. (2012). Planning as inference. Trends Cogn. Sci. 16, 485-488.

Botvinick, M.M., and Cohen, J.D. (2014). The computational and neural basis of cognitive control: charted territory and new frontiers. Cogn. Sci. 38, 1249-1285.

Botvinick, M.M., Niv, Y., and Barto, A.C. (2009). Hierarchically organized behavior and its neural foundations: A reinforcement-learning perspective. Cognition 113, 262-280.

Butler, W.N., Hardcastle, K., and Giocomo, L.M. (2019). Remembered reward locations restructure entorhinal spatial maps. Science 363, 1447-1452.

Carpenter, F., Manson, D., Jeffery, K., Burgess, N., and Barry, C. (2015). Grid cells form a global representation of connected environments. Curr. Biol. CB 25, 1176-1182.

Cohen, J.D., Dunbar, K., and McClelland, J.L. (1990). On the control of automatic processes: a parallel distributed processing account of the Stroop effect. Psychol. Rev. 97, 332-361.

Constantinescu, A.O., O'Reilly, J.X., and Behrens, T.E.J. (2016). Organizing conceptual knowledge in humans with a gridlike code. Science 352, 1464-1468.

Corbit, L.H., and Balleine, B.W. (2016). Learning and Motivational Processes Contributing to PavlovianInstrumental Transfer and Their Neural Bases: Dopamine and Beyond. In Behavioral Neuroscience of Motivation, E.H. Simpson, and P.D. Balsam, eds. (Cham: Springer International Publishing), pp. 259-289.

Corbit, L.H., Janak, P.H., and Balleine, B.W. (2007). General and outcome-specific forms of Pavlovianinstrumental transfer: the effect of shifts in motivational state and inactivation of the ventral tegmental area. Eur. J. Neurosci. 26, 3141-3149.

Cushman, F., and Morris, A. (2015). Habitual control of goal selection in humans. Proc. Natl. Acad. Sci. U. S. A. 112, 13817-13822.

Daw, N.D., and Dayan, P. (2014). The algorithmic anatomy of model-based evaluation. Philos. Trans. R. Soc. Lond. B. Biol. Sci. 369.

Daw, N.D., Niv, Y., and Dayan, P. (2005). Uncertainty-based competition between prefrontal and dorsolateral striatal systems for behavioral control. Nat. Neurosci. 8, 1704-1711.

Daw, N.D., Gershman, S.J., Seymour, B., Dayan, P., and Dolan, R.J. (2011). Model-based influences on humans' choices and striatal prediction errors. Neuron 69, 1204-1215.

Dayan, P. (1993). Improving Generalization for Temporal Difference Learning: The Successor Representation. Neural Comput. 5, 613-624.

Derdikman, D., Whitlock, J.R., Tsao, A., Fyhn, M., Hafting, T., Moser, M.-B., and Moser, E.I. (2009). Fragmentation of grid cell maps in a multicompartment environment. Nat. Neurosci. 12, 1325-1332.

Dezfouli, A., and Balleine, B.W. (2012). Habits, action sequences and reinforcement learning. Eur. J. Neurosci. 35, 1036-1051.

Dickinson, A., and Balleine, B. (1994). Motivational control of goal-directed action. Anim. Learn. Behav. 22, 1-18. 
Dickinson, A., and Balleine, B.W. (2002). The role of learning in motivation. In Volume 3 of Steven's Handbook of Experimental Psychology: Learning, Motivation, and Emotion, C.R. Gallistel, ed. (New York: Wiley), pp. 497-533.

Dordek, Y., Soudry, D., Meir, R., and Derdikman, D. (2016). Extracting grid cell characteristics from place cell inputs using non-negative principal component analysis. ELife 5, e10094.

Everitt, B.J., and Robbins, T.W. (2016). Drug Addiction: Updating Actions to Habits to Compulsions Ten Years On. Annu. Rev. Psychol. 67, 23-50.

Gershman, S.J. (2018). The Successor Representation: Its Computational Logic and Neural Substrates. J. Neurosci. 38, 7193-7200.

Gershman, S., and Wilson, R. (2010). The Neural Costs of Optimal Control. In Advances in Neural Information Processing Systems 23, J.D. Lafferty, C.K.I. Williams, J. Shawe-Taylor, R.S. Zemel, and A. Culotta, eds. (Curran Associates, Inc.), pp. 712-720.

Gillan, C.M., Kosinski, M., Whelan, R., Phelps, E.A., and Daw, N.D. (2016). Characterizing a psychiatric symptom dimension related to deficits in goal-directed control. ELife 5.

Gustafson, N.J., and Daw, N.D. (2011). Grid Cells, Place Cells, and Geodesic Generalization for Spatial Reinforcement Learning. PLOS Comput. Biol. 7, e1002235.

Hafting, T., Fyhn, M., Molden, S., Moser, M.-B., and Moser, E.I. (2005). Microstructure of a spatial map in the entorhinal cortex. Nature 436, 801-806.

He, Q., and Brown, T.I. (2019). Environmental Barriers Disrupt Grid-like Representations in Humans during Navigation. Curr. Biol. CB 29, 2718-2722.e3.

Huys, Q.J.M., and Renz, D. (2017). A Formal Valuation Framework for Emotions and Their Control. Biol. Psychiatry 82, 413-420.

Huys, Q.J.M., Lally, N., Faulkner, P., Eshel, N., Seifritz, E., Gershman, S.J., Dayan, P., and Roiser, J.P. (2015). Interplay of approximate planning strategies. Proc. Natl. Acad. Sci. U. S. A. 112, 3098-3103.

Jang, J.-S., Lee, S.-Y., and Shin, S.-Y. (1988). An Optimization Network for Matrix Inversion. pp. 397-401.

Kappen, H.J. (2005). Linear theory for control of nonlinear stochastic systems. Phys. Rev. Lett. 95, 200201. Keramati, M., Dezfouli, A., and Piray, P. (2011). Speed/accuracy trade-off between the habitual and the goal-directed processes. PLoS Comput. Biol. 7, e1002055.

Keramati, M., Smittenaar, P., Dolan, R.J., and Dayan, P. (2016). Adaptive integration of habits into depthlimited planning defines a habitual-goal-directed spectrum. Proc. Natl. Acad. Sci. U. S. A. 113, 1286812873.

Kool, W., and Botvinick, M. (2018). Mental labour. Nat. Hum. Behav. 2, 899-908.

Kool, W., McGuire, J.T., Rosen, Z.B., and Botvinick, M.M. (2010). Decision making and the avoidance of cognitive demand. J. Exp. Psychol. Gen. 139, 665-682.

Krebs, R.M., Boehler, C.N., and Woldorff, M.G. (2010). The influence of reward associations on conflict processing in the Stroop task. Cognition 117, 341-347.

Kurzban, R., Duckworth, A., Kable, J.W., and Myers, J. (2013). An opportunity cost model of subjective effort and task performance. Behav. Brain Sci. 36.

Lehnert, L., Tellex, S., and Littman, M.L. (2017). Advantages and Limitations of using Successor Features for Transfer in Reinforcement Learning. ArXiv170800102 Cs Stat.

Louie, K., Grattan, L.E., and Glimcher, P.W. (2011). Reward value-based gain control: divisive normalization in parietal cortex. J. Neurosci. Off. J. Soc. Neurosci. 31, 10627-10639.

Mahadevan, S. (2012). Representation Policy Iteration. ArXiv12071408 Cs.

Mahadevan, S., and Maggioni, M. (2007). Proto-value Functions: A Laplacian Framework for Learning Representation and Control in Markov Decision Processes. J. Mach. Learn. Res. 8, 2169-2231.

Mattar, M.G., and Daw, N.D. (2018). Prioritized memory access explains planning and hippocampal replay. Nat. Neurosci. 21, 1609-1617.

Miller, K.J., Shenhav, A., and Ludvig, E.A. (2019). Habits without values. Psychol. Rev. 126, 292-311. 
Momennejad, I., Russek, E.M., Cheong, J.H., Botvinick, M.M., Daw, N.D., and Gershman, S.J. (2017). The successor representation in human reinforcement learning. Nat. Hum. Behav. 1, 680-692.

Russek, E.M., Momennejad, I., Botvinick, M.M., Gershman, S.J., and Daw, N.D. (2017). Predictive representations can link model-based reinforcement learning to model-free mechanisms. PLoS Comput. Biol. 13, e1005768.

Sanguinetti-Scheck, J.I., and Brecht, M. (2019). Home, head direction stability and grid cell distortion. BioRxiv 602771.

Shenhav, A., Musslick, S., Lieder, F., Kool, W., Griffiths, T.L., Cohen, J.D., and Botvinick, M.M. (2017). Toward a Rational and Mechanistic Account of Mental Effort. Annu. Rev. Neurosci. 40, 99-124.

Solstad, T., Boccara, C.N., Kropff, E., Moser, M.-B., and Moser, E.I. (2008). Representation of geometric borders in the entorhinal cortex. Science 322, 1865-1868.

Stachenfeld, K.L., Botvinick, M.M., and Gershman, S.J. (2017). The hippocampus as a predictive map. Nat. Neurosci. 20, 1643-1653.

Sutton, R.S. (1995). TD Models: Modeling the World at a Mixture of Time Scales. In Machine Learning Proceedings 1995, A. Prieditis, and S. Russell, eds. (San Francisco (CA): Morgan Kaufmann), pp. 531-539.

Sutton, R.S., and Barto, A.G. (2018). Reinforcement Learning: An Introduction (Cambridge, Massachusetts: MIT Press).

Sutton, R.S., and Pinette, B. (1985). The learning of world models by connectionist networks. In Seventh Annual Conference of the Cognitive Science Society, pp. 54-64.

Teh, Y., Bapst, V., Czarnecki, W.M., Quan, J., Kirkpatrick, J., Hadsell, R., Heess, N., and Pascanu, R. (2017). Distral: Robust multitask reinforcement learning. In Advances in Neural Information Processing Systems 30, I. Guyon, U.V. Luxburg, S. Bengio, H. Wallach, R. Fergus, S. Vishwanathan, and R. Garnett, eds. (Curran Associates, Inc.), pp. 4496-4506.

Todorov, E. (2007). Linearly-solvable Markov decision problems. In Advances in Neural Information Processing Systems 19, B. Schölkopf, J.C. Platt, and T. Hoffman, eds. (MIT Press), pp. 1369-1376.

Todorov, E. (2008). General duality between optimal control and estimation. In 200847 th IEEE Conference on Decision and Control, pp. 4286-4292.

Todorov, E. (2009). Efficient computation of optimal actions. Proc. Natl. Acad. Sci. U. S. A. 106, 1147811483.

Tolman, E.C. (1948). Cognitive maps in rats and men. Psychol. Rev. 55, 189-208.

Tolman, E.C., and Gleitman, H. (1949). Studies in learning and motivation; equal reinforcements in both end-boxes; followed by shock in one end-box. J. Exp. Psychol. 39, 810-819.

Westbrook, A., Kester, D., and Braver, T.S. (2013). What Is the Subjective Cost of Cognitive Effort? Load, Trait, and Aging Effects Revealed by Economic Preference. PLOS ONE 8, e68210.

Whittington, J.C., Muller, T.H., Mark, S., Chen, G., Barry, C., Burgess, N., and Behrens, T.E. (2019). The Tolman-Eichenbaum Machine: Unifying space and relational memory through generalisation in the hippocampal formation. BioRxiv 770495.

Wimmer, G.E., and Shohamy, D. (2012). Preference by association: how memory mechanisms in the hippocampus bias decisions. Science 338, 270-273.

de Wit, S., Niry, D., Wariyar, R., Aitken, M.R.F., and Dickinson, A. (2007). Stimulus-outcome interactions during instrumental discrimination learning by rats and humans. J. Exp. Psychol. Anim. Behav. Process. 33, $1-11$.

Woodford, M. (2012). Prospect Theory as Efficient Perceptual Distortion. Am. Econ. Rev. 102, 41-46. Zorowitz, S., Momennejad, I., and Daw, N.D. (2019). Anxiety, avoidance, and sequential evaluation. BioRxiv 724492. 